\title{
Improving Efficiency by Thermodynamic and Gravitational Cycle
}

\author{
Zhang Quan \\ Shanghai Kongtai Energy Techonolgy Co., Ltd, Shanghai, China \\ Email: CAIDCOM@hotmail.com
}

How to cite this paper: Quan, Z. (2017) Improving Efficiency by Thermodynamic and Gravitational Cycle. Energy and Power Engineering, 9, 250-260. https://doi.org/10.4236/epe.2017.94B030

Received: February 16, 2017

Accepted: March 30, 2017

Published: April 6, 2017

\begin{abstract}
The efficiency of Rankine cycle and its derivative cycles are severely affected by droplets condensed in the process of vapor expansion, which not only limit its maximum efficiency, but also cause extremely low efficiency around 3\% when using low grade heat source. This paper introduces a new theory of Thermodynamic and Gravitational Cycle to explain the concept of MLC, and analyses the reasons MLC OTEC cannot be realized till now. Then The concept of Thermodynamic and Gravitational Closed-Cycle (TGCC) to overcome disadvantage of Rankine and MLC cycle are proposed, and its especial cycle process and efficiency model in detail are discussed. And then we propose a method of combining vapor with mist lift to improve efficiency further, and analyze the new ideal efficiency model (a maximum up to 18.17\%) using carbon dioxide sample, indicate the dryness of liquid-vapor mixture is the key factor to improve efficiency. In conclusion, TGCC with mist lift has the potential to significantly improve efficiency and reduce the cost of electricity produced from low grade heat source, such as OTEC and industrial waste heat.
\end{abstract}

\section{Keywords}

Thermodynamic and Gravitational Cycle, Mist Lift, Height, Droplet, Low Grade Heat Source

\section{Introduction}

In the field of heat engine, Carnot Cycle and Rankine Cycle are the most important theories guiding us to study thermal machine and its efficiency since they were established in 19th century. The Carnot cycle is a theoretical construct about thermodynamic cycle which provides an upper limit on the efficiency that any classical thermodynamic engine can achieve during the conversion of heat into work, or conversely, the efficiency of a refrigeration system. The Rankine 
cycle is an idealized thermodynamic cycle of a heat engine that converts heat into mechanical work. And, the Rankine cycle is the standard model used to predict the performance of steam turbine systems. Here are many factors influencing the efficiency of the real Rankine Cycle, such as the temperature of steam into the superheat region, the temperature of condensing, the dryness and droplets carried by the steam.

In particular the efficiency of the steam turbine will be limited by water droplet formation [1]. As the water condenses, water droplets hit the turbine blades at high speed causing pitting and erosion, gradually decreasing the life of turbine blades and efficiency of the turbine. Obviously, the efficiency of Rankine Cycle and its derivative cycles are severely affected by droplets condensed in the process of vapor expansion, which not only limit its maximum efficiency, but also cause extremely low efficiency when used in low grade heat source, such as the best efficiency of OTEC (Ocean Thermal Energy Conversion) cycle is around $3 \%$.

On the other hand, a proper condensation proportion of steam is beneficial to improve thermal efficiency. For example, most enthalpy drop comes from the heat of steam condensing in the last stage of multi-stage steam turbine, the condensation heat from $10 \%$ steam accounted for $79 \%$ of the total enthalpy drop [2]. Thus, the largest humidity of exhaust steam is suggested $10 \%-12 \%$. Obviously, the condensation heat of steam is beneficial to improving thermal efficiency, while droplets are very harmful to turbine life and its efficiency. Therefore, most scientists focused on studying how to control dryness of steam and the liquid-vapor two phase flow [1] [2].

With great different from previous studies, this paper establishes and proposes a new theory named Thermodynamic and Gravitational Cycle (TGC), in order to take full advantages of condensation heat and carrying capability of steam. TGC theory can significantly improve efficiency of energy conversion, and significantly reduce the cost of electricity produced from low grade heat source, such as ocean water and industrial waste heat.

\section{The TGC Theory}

\subsection{The TGC Concept}

Rankine Cycle and its derivative cycles belong to pure thermodynamic cycle. The earth gravity has no influence on those thermodynamic cycles. On the contrary, if the earth gravity and heat plays a vital role in the conversion process of energy, the conversion cycle is called Thermodynamic and Gravitational Cycle (TGC).

TGC phenomena are common in natural world. For example, water evaporates on the earth surface and rises to upper air due to heated by sun; water steam condenses and forms a cloud while rising; then cloud rains and drop to the earth surface when meeting cold air. The rainwater fallen in highland or mountain is the source to produce hydroelectric power. We can also find TGC phenomenon in some artificiality, such as gravity assisted heat pipe, and MLC 
(Mist Lift Cycle) experiment devices [3].

This paper will discuss two kinds of TGC, i.e. Thermodynamic and Gravitational Open-Cycle (TGOC) and Thermodynamic and Gravitational ClosedCycle (TGCC) to develop the TGC theory.

\subsection{The TGOC Concept}

In particular, MLC is an open-cycle OTEC concept originally developed by Dr. Stuart Ridgway in 1970s [3]. MLC uses a finely perforated titanium plate (mist generator) at the bottom of vacuum chamber. Warm seawater is introduced into the vacuum chamber through $0.1 \mathrm{~mm}$ diameter holes in the titanium plate. The low pressure in the vacuum chamber is maintained such that some of the water flash evaporates-creating a fine mist. Farther up the column, cold seawater is injected into the chamber. The cold seawater lowers the pressure in the top of the chamber. This creates a pressure gradient that drives the mist from the bottom of the chamber to the top. The vapor component of the mist is completely condensed into the cold water by the time the lift is complete. The lift height is such that water is lifted above sea level, and then the water is drained into the ocean.

Obviously, MLC is very different from Rankine Cycle. MLC introduced gravitation to thermodynamic open-cycle. That can be explained by the concept of Thermodynamic and Gravitational Open-Cycle (TGOC) whose process theory is shown in Figure 1 comparing with Rankine Cycle.

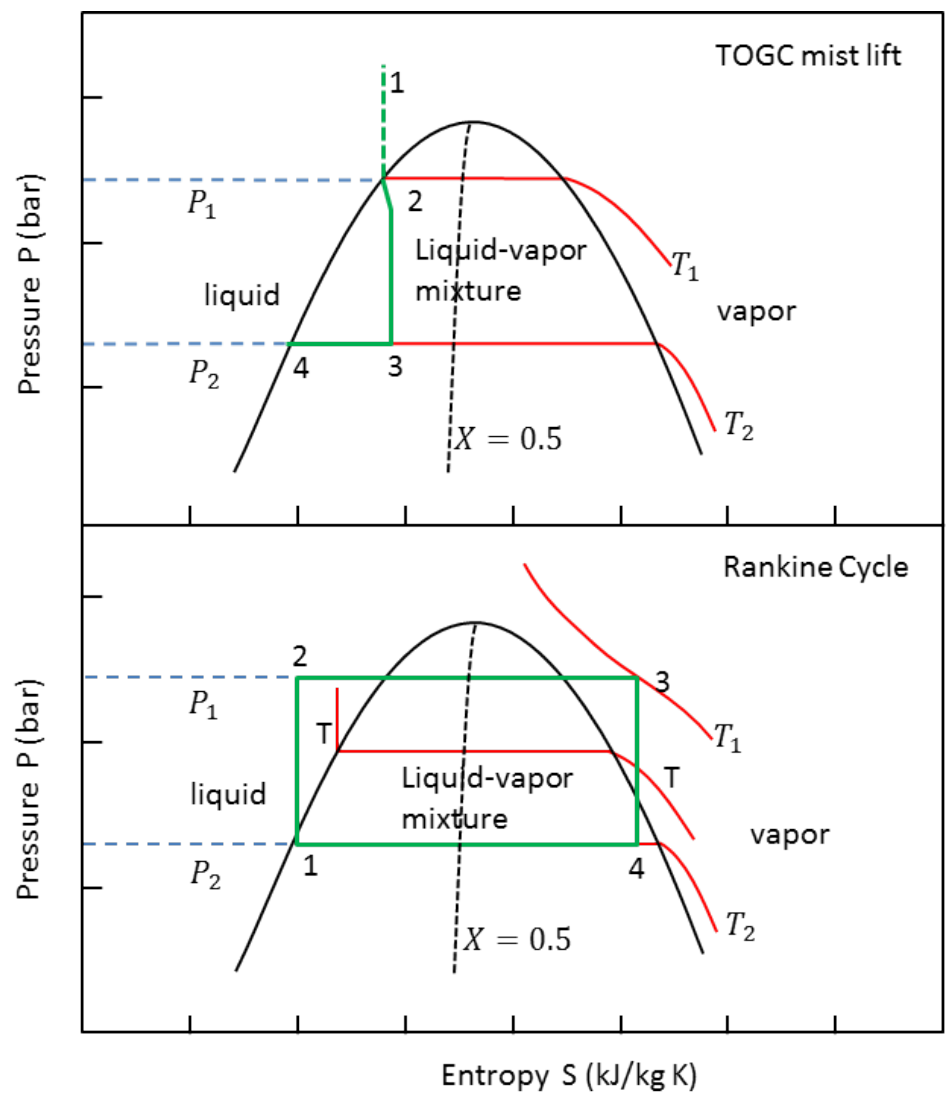

Figure 1. MLC and Rankine cycle's P-S diagram. 
As we know, Rankine is a close cycle with 4 typical stages shown in Figure 1. The stage 1 - 2: The working fluid is pumped from low to high pressure. As the fluid is a liquid at this stage, the pump requires little input energy. The stage 2 3: The high pressure liquid enters a boiler where it is heated at constant pressure by an external heat source to become a dry saturated vapor. The stage $3-4$ : The dry saturated vapor expands through a turbine, generating power. This decreases the temperature and pressure of the vapor, and some condensation may occur. The stage $4-1$ : The wet vapor then enters a condenser where it is condensed at a constant pressure to become a saturated liquid.

Fundamentally different from Rankine cycle, the concept of TOGC mist lift has 3 primary stages shown in Figure 1. The stage 1 - 2: warm seawater from sea surface get a high pressure at bottom of vacuum chamber, seawater is injected into low pressure vacuum chamber through the mist generator. Here is a very large pressure drop. The stage 2 - 3: the mist contains a mass of droplets and some vapor generated by flash evaporation; vapor expands through the vacuum chamber, and lift droplets to the top. The stage 3 - 4: The vapor component of the mist is completely condensed into cold water by cooler spraying water at the top.

So, MLC uses little vapor expansion and condensation to lift mass of droplets, while Rankine Cycle uses dry vapor expansion to drive steam turbine. Wet vapor with small dryness can lift to a high altitude, which is the key to develop the theory of TGC. Although the thermodynamic feasibility of mist lift has been established based on much experiments and researches [5]-[11], its characteristics are poorly understood and there is no sound theoretical support. The most critical issue of OTEC MLC is ignored till now, i.e. without heat exchanger, the temperature difference $(17 \mathrm{~K})$ is not enough to lift all warm seawater introduced into vacuum chamber. As we know, the latent heat of water evaporation is 2500 $\mathrm{kJ} / \mathrm{kg}$, while the specific isobar heat of water is $4.2 \mathrm{~kJ} / \mathrm{kg} \mathrm{K}$. One unit mass of water evaporation need the heat of 35.3 unit mass of seawater with $17 \mathrm{~K}$ drop of temperature. Obviously, the dryness of mist $(X<0.028)$ is extremely small. Although some researches show that it is realizable to lift 10.0 unit mass of mist to high altitude using 1 unit mass of vapor if provide a much bigger inlet pressure [7]. It is indeed impossible to lift the 33.5 unit mass of mist using 1 unit of steam. That is the reason MLC haven't been realized in OTEC till now from the opinion of this paper. MLC should be realized with higher temperature difference.

In order to avoid the above weaknesses of Rankine Cycle, MLC (TGOC) and other conventional thermodynamic cycles, this paper introduce a better TGC theory, i.e. thermodynamic and Gravitational Closed-Cycle (TGCC), and then combine it with mist lift.

\subsection{The TGCC Concept}

TGCC is a completely closed thermodynamics and gravitational cycle with completely recycling working medium.

A proposed TGCC schematic diagram for OTEC is shown in Figure 2, working 


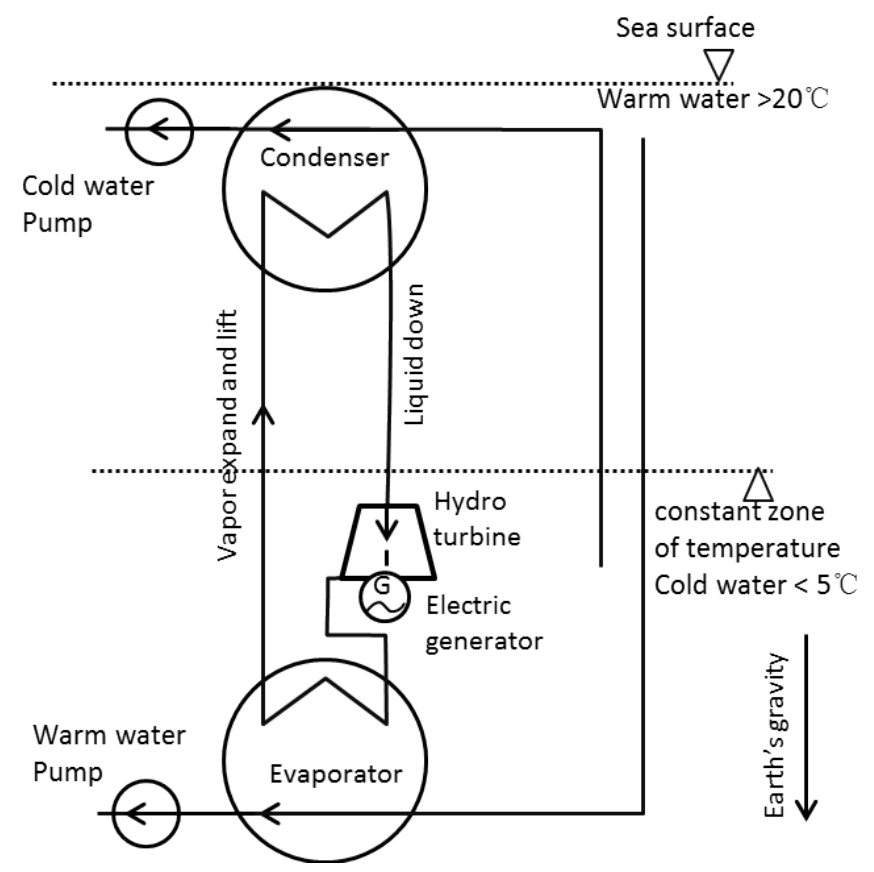

Figure 2. The schematic diagram of TGCC for OTEC.

medium is heated and evaporated into vapor in evaporator under high pressure and temperature condition, then vapor expand and lift up against gravity to the top condenser. Vapor is completely condensed to cold liquid by lower temperature condenser and that continuously produce lower pressure. Cold liquid is continuously collected into a reservoir and keep the reservoir full, while the cold liquid down to the hydro turbine due to the gravitational force. Liquid gravitational potential energy is conversed to mechanical energy, and then conversed to electric energy.

\subsection{TGCC Process and Efficiency}

The process of TGCC cycle contains 5 stages shown in Figure 3.

- The stage 1 - 2: Cold liquid is heated at constant pressure $p_{1}$ by an external heat source to become a saturated vapor at bottom. The vapor temperature is $T_{1}$, the vapour specific enthalpy is $H_{1}$, the quantity of heat absorbed from external heat resource is $Q_{x}$.

- The stage 2 - 3: The saturated vapor expands and lifts through the lifting pipe to the top against gravity. This decreases the temperature and pressure of the vapor, and some condensation may occur. Here the vapor temperature is $T_{2}$, the vapor pressure is $p_{2}$, and the specific enthalpy of vapor is $H_{2}$.

- The stage 3 - 4: The wet vapor then enters a condenser where it is condensed at the constant pressure $p_{2}$ to become a saturated liquid with the same temperature $T_{2}$. Here the specific enthalpy of liquid is $H_{3}$, the quantity of heat released to external cold resource is $Q_{f}$.

- The stage 4 - 5: The liquid flow down in the declining pipe to the bottom due to gravitational force, while liquid pressure becomes higher and gets the maximum at the point of hydro turbine. Liquid temperature $T_{2}$ and specific 


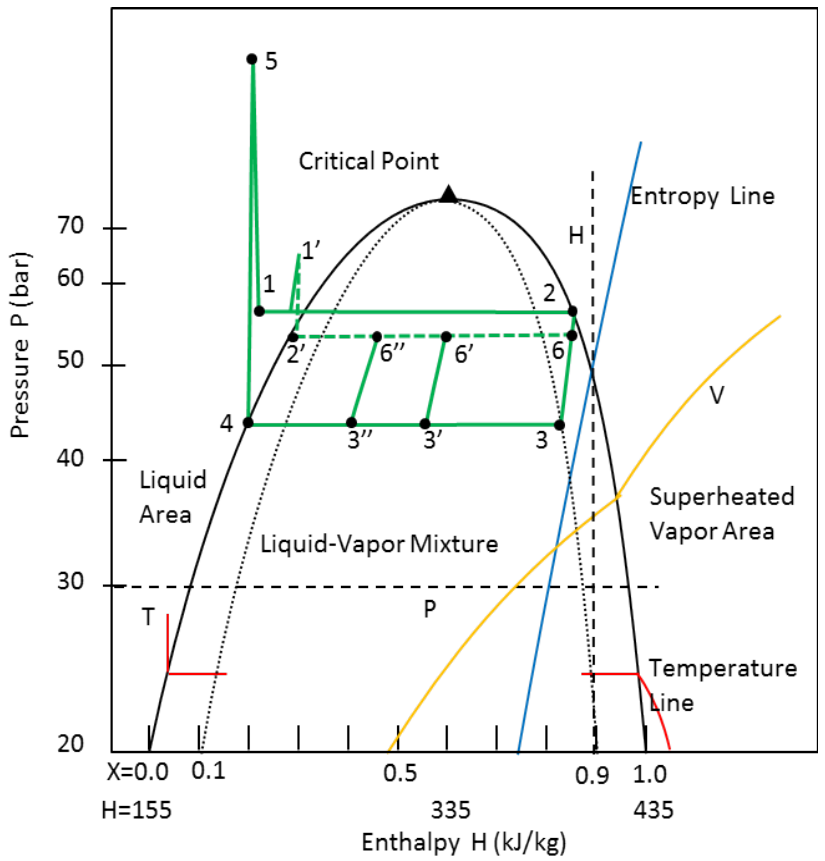

Figure 3. The P-H diagram of TGCC.

enthalpy $\mathrm{H}_{3}$ is almost invariant.

- The stage 5 - 1: The liquid in declining pipe is drained into evaporator through hydro turbine. Liquid temperature $T_{2}$ and specific enthalpy $H_{3}$ is almost invariant. But the liquid pressure is plunged from $p_{4}$ to $p_{1}$. The output work $W_{g}$ of liquid almost equals to the produced electric energy.

In the stage $2-3$, the vapor pressure vary with the lift height, its equation is

$$
p_{2}=p_{1} \cdot \mathrm{e}^{h g M /(1000 \cdot T \cdot N \cdot k)},
$$

Herein, $p_{1}$ is the original pressure at bottom $(h=0), p_{2}$ is the pressure at the height of $h, e$ is the natural constant, $g$ is the acceleration of gravity, $M$ is molar mass of gasmolecular, $T$ is the degree kelvin $(K), N$ is the Avogadro constant, and $k$ is the Boltzmann constant.

In the stage $2-3$, the vapor temperature also vary with the lift height, its temperature drop equation without condensation is:

$$
\Delta T=-\frac{g}{C_{p}} \Delta h
$$

Its temperature drop equation with condensation is:

$$
\Delta T_{s}=-\frac{g}{C_{p}} \Delta h+\frac{L}{C_{p}} \Delta q
$$

Herein, $\Delta T$ is the degree of temperature drop, $g$ is acceleration of gravity, $C_{p}$ is specific isobaric heat capacity, $\Delta h$ is the lift height, $L$ is latent heat, $\Delta q$ is the mass of condensation. Normally, the temperature drop values comply with the sequence $\left|\Delta T_{s}\right|<|\Delta T|<\left|T_{1}-T_{2}\right|$.

According to the cycle process above, we can get its theoretic efficiency equation: 


$$
\eta=1-\frac{Q_{f}}{Q_{x}}=\frac{H_{1}-H_{2}}{H_{1}-H_{3}}
$$

Herein, $H_{1}$ is specific enthalpy of saturated vapour, $H_{2}$ is specific enthalpy of wet vapour lifted to the top, $H_{3}$ is specific enthalpy of condensed liquid.

Accordingly, its ideal efficiency depends on the lift height:

$$
\eta_{h}=\frac{V\left(\rho g h+p_{2}-p_{1}\right)}{m\left(H_{1}-H_{3}\right)}
$$

Herein, $V$ the volume of liquid, $\rho$ is density of liquid, $m=\rho V$ is the mass of liquid (or vapour), $g$ is acceleration of gravity, $h$ is the lift height, $p_{1}$ is the vapour pressure at bottom, $p_{2}$ is the vapour pressure at top.

According to the Equations (1) (2) and (3), the lift height $h$ is limited by the temperature difference $T_{1}-T_{2}$. For the example, given $T_{1}=20^{\circ} \mathrm{C}, T_{2}=10^{\circ} \mathrm{C}$, then the maximum condensation height of carbon dioxide gas is 1360 meters. Otherwise, higher than 1360 meters, the carbon dioxide will not condensate at the same temperature $T_{2}$ since pressure will become lower with higher lift height. Table 1 show detail attributes and parameters of carbon dioxide sample.

According to the Equation (5) and Table 1, we can get the maximum efficiency $\eta_{h} \cong 5.08 \%$ that seems to be a little better comparing with conversional OTEC efficiency (around 3\%). Considering building a TGCC pipe system reach up to the height of 1360 meters is extremely hard and expensive. If the real lift height is reduced to 600 meters, the efficiency $\eta_{h}$ is less than $3 \%$. Thus, the efficiency still need be improved further in order realize more economic and practical.

Reducing the height will decrease efficiency, while vapour condensation and mist lift will be easier at the same cooling temperature. That gives a potential way to improve efficiency at a feasible height. So, this paper combine mist lift concept to TGCC theory to significantly improve its efficiency.

\subsection{Method of Improving TGCC Efficiency}

Add a mist generator above the evaporator, we can get a new process of TGCC

Table 1. Attributes and parameters of carbon dioxide.

\begin{tabular}{ccccc}
\hline Item & $\begin{array}{c}\text { Vapor in } \\
\text { lifting pipe } \\
\text { Bottom }\end{array}$ & $\begin{array}{c}\text { Vapor in lifting } \\
\text { pipe Top }\end{array}$ & $\begin{array}{c}\text { Liquid in } \\
\text { declining } \\
\text { pipe Bottom }\end{array}$ & Unit \\
\hline High of Lift: & 0 & 1360 & $(1360)$ & meters \\
Pressure: & 57.3 & 45.0 & 171.6 & $\mathrm{bar}$ \\
Condensing Temperature: & 20.0 & 10.0 & -- & Celsius \\
Specific Enthalpy fluid: & 255.8 & 225.7 & 225.7 & $\mathrm{~kJ} / \mathrm{kg}$ \\
Specific Enthalpy gas: & 407.9 & 422.9 & -- & $\mathrm{kJ} / \mathrm{kg}$ \\
$\begin{array}{c}\text { Specific isobar heat capacity } \\
\text { fluid: }\end{array}$ & 4.266 & 2.996 & 2.996 & $\mathrm{~kJ} / \mathrm{kg} \mathrm{K}$ \\
Specific isobar heat capacity & & & & $\mathrm{kJ} / \mathrm{kg} \mathrm{K}$ \\
gas: & 4.574 & 2.577 & - & \\
\hline
\end{tabular}


cycle contains 8 stages shown in Figure 3.

- The stage 1 - 2: Cold liquid of $m_{1}$ mass is heated at constant pressure $p_{1}$ by an external heat source to become a saturated vapor at bottom. The vapor temperature is $T_{1}$, the vapour specific enthalpy is $H_{1}$, the quantity of heat absorbed from external heat resource is $Q_{x}$. Saturated vapor dryness is near to 1.0 .

- The stage 1-1'-2': Cold liquid of $m_{2}$ mass is heated at constant pressure $p_{1}$, then is pumped into mist generator with a higher pressure (point 1'), and then is sprayed at a lower pressure (point 2'). Mist is produced at point 2', mist dryness is near to 0 .

- The stage 2 - 6: The saturated vapor expands and lifts to the height where mist generator installed. Vapor flow already gets a very fast velocity.

- The stage $(2$ ', 6)-6': Vapor meet with mist, and then combine into liquid-vapor mixture at point 6 . Its mass is $\left(m_{1}+m_{2}\right)$.

- The stage 6'-3': the vapor expands through the lifting pipe and lift droplets to the top condenser. This decreases the temperature and pressure of the vapor, and some condensation may occur. The dryness of the mixture will be smaller.

- The stage 3'-4: The mixture enters a condenser where vapor is condensed at the constant pressure $p_{2}$. The mixture completely become a saturated liquid at temperature $T_{2}$. Here the specific enthalpy of liquid is $H_{3}$, the quantity of heat released to external cold resource is $Q_{f}$.

- The stage 4 - 5: The liquid of $\left(m_{1}+m_{2}\right)$ mass flows in the declining pipe down to the bottom due to gravitational force, while liquid pressure becomes higher and gets the maximum at the point of hydro turbine. Liquid temperature $T_{2}$ and specific enthalpy $H_{3}$ is almost invariant.

- The stage 5 - 1: The liquid in declining pipe is drained into evaporator through hydro turbine. Liquid temperature $T_{2}$ and specific enthalpy $H_{3}$ is almost invariant. But the liquid pressure is plunged from $p_{4}$ to $p_{1}$. The output work $W_{g}$ of liquid almost equals to the produced electric energy.

Since a mass of $m_{2}$ mist is added into vapor of $m_{1}$, the ideal efficiency is different from Equation (5), its new equation is as below:

$$
\eta_{h}=\frac{V\left(\rho g h+p_{2}-p_{1}\right)}{m_{1}\left(H_{1}-H_{3}\right)+m_{2} C_{p} \Delta T}
$$

Herein, $V$ the liquid volume, $\rho$ is the density of liquid, $\left(m_{1}+m_{2}\right)=\rho V$ is the mass of all working medium, $g$ is acceleration of gravity, $h$ is the lift height, $p_{1}$ is the vapour pressure at bottom, $p_{2}$ is the vapour pressure at top, $H_{1}$ is specific enthalpy of saturated vapour, $H_{3}$ is specific enthalpy of condensed liquid. $C_{p}$ is the specific isobar heat capacity of liquid, $\Delta T$ is the temperature rise. $m_{2} C_{p} \Delta T$ is the heat absorbed by the liquid for producing mist.

If $m_{2}=m_{1}$, the dryness of mixture is $X \cong 0.50$. According to Equation (6) and the parameters in Table 1 , the ideal maximum efficiency is $\eta_{h}=8.55 \%$ $8.55 \%$. 
If $m_{2}=2 m_{1}$, the dryness of mixture is $X \cong 0.33$. The maximum efficiency will be $\eta_{h}=11.06 \% 11.06 \%$.

And if $m_{2}=8 m_{1}$, The dryness of mixture is $X \cong 0.11$, the maximum efficiency will be $\eta_{h}=18.17 \% 18.17 \%$, which is an exciting value comparing with conventional OTEC efficiency ( $3 \%-4.5 \%)$. Even we reduce the lift height to the half $(680 \mathrm{~m}), \quad \eta_{h}$ is still larger than $9 \%$.

In the condition supposed this paper, efficiency increases with more and more mist combined, at the same time dryness decreases accordingly. We can get the maximum efficiency line as shown in Figure 4. Obviously, to left the cycle efficiency trends to a closed MLC without vapor, and to right the cycle efficiency trends to a closed Rankine cycle without mist.

One the one hand, combining more mist with vapour is the primary method to improve efficiency significantly, which will move the stage of $6^{\prime}-3^{\prime}$ to a new position (6"-3") trending to zero dryness as shown in Figure 3.

On the other hand, Mist Lift is significantly influenced by the diameter of mist droplets. Many institutes such as the University of Virginia, Dartmouth College and Solar Energy Research Institute have finished much experiments and researches [7]-[12] on mist lift flow rate, velocity, droplet diameter, and inlet pressure, which give full evidences and summarize laws of mist lift. Experiments show that inlet pressure influences mist lift performance [7]. Bigger inlet pressure can get a bigger maximum flow rate before rainout appears. If inlet pressure is $0.2 \mathrm{bar}$, then the maximum flow rate is $181 \mathrm{~kg} / \mathrm{s}$ without rainout, dryness is 0.22 . If inlet pressure is $5.0 \mathrm{bar}$, then the maximum flow rate is $945 \mathrm{~kg} / \mathrm{s}$ without rainout, the dryness is about 0.09 . Figure 5 shows the droplet velocity and temperature changing rules in mist lift stage [7]. Droplets velocity can be accelerated by the vapor expanding at bottom and condensing at top. Figure 5 shows droplets

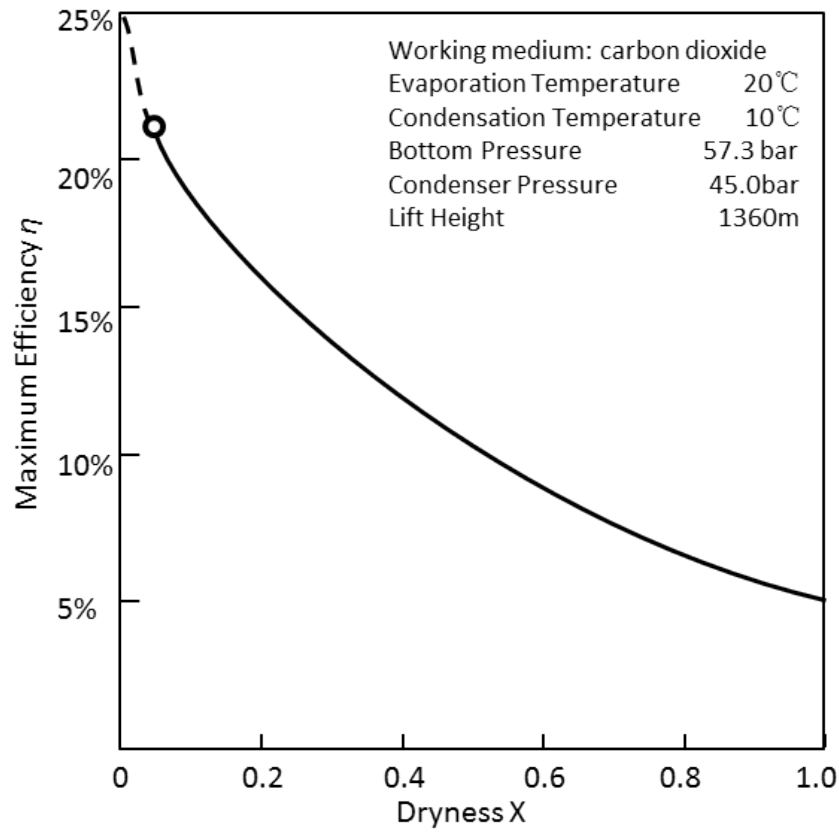

Figure 4. Maximum efficiency line of TGCC. 


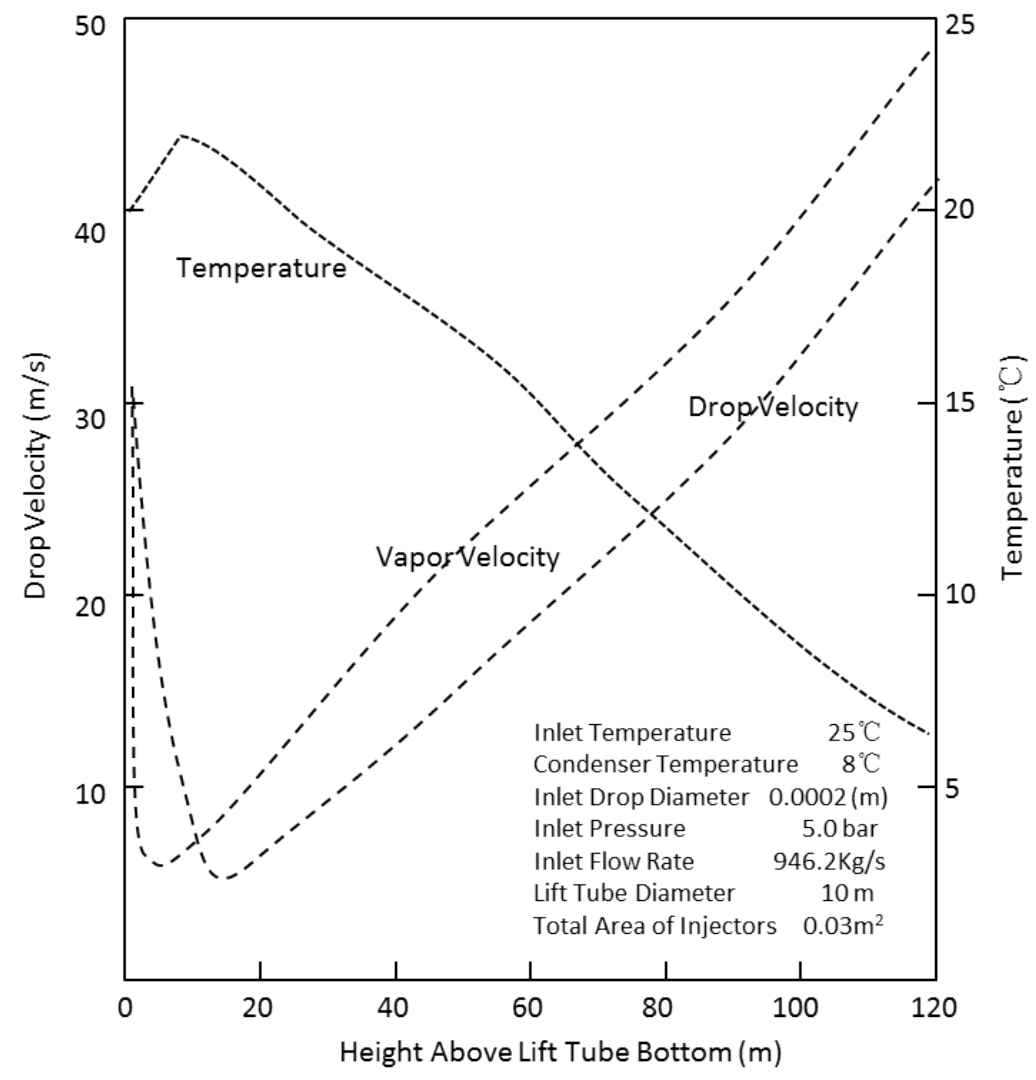

Figure 5. Sample outputs from single-group models.

get the maximum velocity at top end of the short lift pipe. We can lengthen the lift pipe to its upper limit so that provide a space for droplet's moderating process, in order to converse all kinetic energy to gravitational potential energy.

\section{Conclusions}

Obviously, thermodynamic and Gravitational Closed-Cycle (TGCC) is completely different with Rankine cycle and other conversional heat engine theory. TGCC is also very different with MLC, MLC depend on injecting mist, and needs no heat exchanger.

TGCC can take full advantage of the condensation during vapor expands and lifts up. The model of TGCC combining with mist lift can significantly improve efficiency of energy process and conversation. For the carbon dioxide case discussed above, with the temperature difference of $10 \mathrm{~K}$, the ideal maximum efficiency may beyond $20 \%$ as the mixture dryness decreased to its minimum possible value. This result value is significantly larger than the efficiency (around 3\%) of conventional OTEC using Rankine cycle. Thus, TGCC theory gives a new direction to reduce the cost of electricity especially produced from low temperature heat source, such as ocean water and industrial waste heat.

TGC or TGCC theory should be taken seriously and be studied further from now on. The major areas of research required to develop TGC include:

- To study the lift capability of liquid-vapor mixture with variant dryness, and 
find the minimum dryness for variant working mediums, such as carbon dioxide, Nitrogen, Freon, water and etc.

- To study the evolution process of the flow velocity of liquid-vapor mixture, since vapor acceleration process and moderating process are influenced by temperatures, lift pipe diameter, dryness, and lift height.

- To select feasible temperature difference and the best lift height of TGCC plant, since either too high or too low will decrease the efficiency.

- To develop better mist generator with narrow spectrum to avoid the disadvantage of large difference of droplets diameter, since multi-group diameter will cause rainout easy.

- Other technologies related with TGC, some are similar the technologies used in OTEC.

\section{References}

[1] Guha, A. (1998) Computation, Analysis and Theory of Two-Phase Flows. The Aeronautical Journal,12, 71-82.

[2] Li, L. and Li, Yu.(2008) Study of Wet Steam Two Phase Flow with Condensation in Steam Turbine. Thermal Turbine, 37, 235-240.

[3] Ridgway, S.L. (1977) The Mist Flow OTEC Plant.Proceedings of 4th OTEC Conference.

[4] Lockheed, M. (2012) Ocean Thermal Energy Conversion Life Cycle Cost Assessment. Bethesda: Lockheed Martin Corporation.

[5] Ridgway,S.L. and Charwat,A.F.(1978) The Design of Laboratory Scale Experiment on Vertical Two Phase Flow with Application to the Mist Flow OTEC Cycle.Proceedings of 5 th OTEC Conference.

[6] Ridgway,S.L.,Lee,C.K.B. and HammondR.P.(1981)Experimental Demonstration of the Feasibility of the Mist Flow Ocean Thermal Enerty Process. Proceedings of 8 th OTEC Conference.

[7] Davenport, R.L. (1980) The Mist Lift Analysis Summary Report. USA Colorado: Solar Energy Research Inst.https://doi.org/10.2172/5112474

[8] Charwat, A.F. (1978) Studies of the Vertical Mist Transport Process for an Ocean Thermal Energy Cycle. SAN-0034-76-1. Los Angeles, CA: University of California.

[9] Ridgway, S.L., Hammond, P.R. (1978) Mist Flow Ocean Thermal Energy Process.RDA-TR-1 07800-002. Marina del Rey, CA: Research and Development Assoc.

[10] Joseph, V.R., Steven, R. and Stuart, R. (2010) Development of Mist Lift: A Cost Breakthrough for OTEC. DE-PS02-09ER09-27.

[11] Wallis,G.B.,Richter,H.J. andBharathan,D. (1979) Analysis of the OTEC Mist Lift Process. SERI-8317-1. Hanover, NH: Thayer School of Engineering, Dartmouth College.

[12] Abbott,C.E. (1977) A Survey of Waterdrop Interaction Experiments. Reviews of Geophysics and Space Physics, 15, 363-374.

https://doi.org/10.1029/RG015i003p00363 
Submit or recommend next manuscript to SCIRP and we will provide best service for you:

Accepting pre-submission inquiries through Email, Facebook, LinkedIn, Twitter, etc. A wide selection of journals (inclusive of 9 subjects, more than 200 journals)

Providing 24-hour high-quality service

User-friendly online submission system

Fair and swift peer-review system

Efficient typesetting and proofreading procedure

Display of the result of downloads and visits, as well as the number of cited articles Maximum dissemination of your research work

Submit your manuscript at: http://papersubmission.scirp.org/

Or contact epe@scirp.org 